\title{
ENTRAMADO CAULINAR EN EPHEDRA TWEEDIANA (EPHEDRACEAE): Una nueVa estrategia de ascenso en Plantas Trepadoras
}

\author{
PABLO A. CABANILLAS ${ }^{1 *}$, M. LUCIA BORNIEGO ${ }^{2}$, ALCIDES A. SAENZ ${ }^{3}$ y JULIO A. \\ HURRELL 4
}

\begin{abstract}
Summary: A new ascent strategy in climbing plants: 'cauline framework' in Ephedra tweediana (Ephedraceae). In this contribution, a novel ascent strategy for the set of climbing plants are described. It has been called 'cauline framework' and has been observed in Ephedra tweediana Fisch. \& C.A. Mey. emend. J.H. Hunz. (Ephedraceae), it was based on different studies in individuals growing in Isla Martín García (Buenos Aires, Argentina). This paper also clarifies the basic biological type of this species, defined as 'liana' (scandent woody plant).
\end{abstract}

Keywords: Climbing plants, ascent strategy, cauline framework, Ephedra tweediana, liana.

\begin{abstract}
Resumen: En esta contribución se describe una estrategia de ascenso novedosa para el conjunto de las plantas trepadoras. Se ha denominado "entramado caulinar" y ha sido observado en Ephedra tweediana Fisch. \& C.A. Mey. emend. J.H. Hunz. (Ephedraceae), a partir de diversos estudios realizados en individuos que crecen en la Isla Martín García (Buenos Aires, Argentina). Asimismo, se clarifica el tipo biológico básico de la especie, definido como "liana" (planta escandente leñosa).
\end{abstract}

Palabras clave: Plantas trepadoras, estrategia de ascenso, entramado caulinar, Ephedra tweediana, liana.

\section{INTRODUCCIÓN}

Un tipo biológico o forma de vida es una categoría morfo-estructural con correlato adaptativo, que se aplica a diversas plantas con independencia de su filiación sistemática. En tanto tipo biológico, las plantas trepadoras carecen de un soporte mecánico autónomo en su estado adulto, es decir no se mantienen erguidas por sí mismas; en su crecimiento, se encaraman sobre distintos soportes

\footnotetext{
1 Cátedra de Morfología Vegetal, Facultad de Ciencias Naturales y Museo, Universidad Nacional de La Plata, Paseo del Bosque s/nro. 1900-La Plata, Argentina. Becario CIC. cabanillaspablo@gmail.com

${ }^{2}$ Cátedra de Morfología Vegetal, FCNyM, UNLP.

3 Instituto de Fisiología Vegetal (INFIVE), Facultad de Ciencias Agrarias y Forestales-FCNyM (UNLP)CONICET, Diag. 113 esq. 61, 1900-La Plata. Becaria CONICET

4 Laboratorio de Etnobotánica y Botánica Aplicada (LEBA), FCNyM, UNLP, Calle 64 nro. 3, 1900-La Plata. Investigador CONICET.
}

(otras plantas o soportes inertes); por lo general, mantienen una conexión permanente con el suelo $\mathrm{y}$, en ausencia de soportes adecuados, presentan vástagos rastreros que pueden devenir trepadores en presencia de aquellos (Hurrell et al., 2011, 2012a). Los mecanismos o estrategias de ascenso son distintas adaptaciones propias de las trepadoras, que les posibilitan crecer en altura; corresponden a estructuras morfológicas diversas que permiten el sostén de la planta al soporte (Font Quer, 1993; Hurrell, 2006; Hurrell et al., 2012a, 2012b; Cabanillas \& Hurrell, 2012).

Lasubclase Gnetidaeesungrupodegimnospermas actuales con particularidades morfológicas tanto vegetativas como reproductivas. Cada uno de los tres órdenes monotípicos que la integran presenta diferentes formas de vida: Gnetales, principalmente lianas y una única especie arbórea (Gnetum gnemon L.); Ephedrales, arbustos xeromórficos erectos, rastreros y trepadores; Welwischiales, un único representante actual (Welwischia mirabilis Hook. f.), xerófita del desierto de Namibia, con un tipo 
biológico de definición discutible (Chase \& Reveal, 2009; Christenhusz et al., 2011).

El género Ephedra está compuesto por unas 40-55 especies distribuidas en la región Mediterránea, sur de Europa y norte de África; en las zonas templadocálidas de Asia y del oeste de Norteamérica, y en el centro-oeste de Sudamérica (Ickert-Bond \& Wojciechowski., 2004; Christenhusz et al., 2011). Ephedra tweediana Fisch. \& C.A. Mey. emend. J.H. Hunz., es una especie distribuida en Brasil austral (Rio Grande do Sul), Uruguay (Río Negro, Flores, Florida, San José, Rocha y Maldonado) y en la Argentina (Salta, Catamarca, Tucumán, Corrientes, Entre Ríos y Buenos Aires). Descrita en diferentes trabajos como una planta leñosa que puede presentar distintos tipos biológicos, los que se hallan representados en la Isla Martín García, donde se realizó el presente estudio (Hunziker, 1949, 1969, 1995; Carlquist, 1996; Hunziker \& Novara, 1998; Ferrucci et al., 2002; Ickert-Bond \& Wojciechowski., 2004; Hurrell, 2006; Giehl, 2012; Instituto de Botánica Darwinion, 2012; Souza, 2012).

La presente contribución tiene por finalidad precisar el tipo biológico trepador de E. tweediana y especificar su estrategia de ascenso, no definida con anterioridad para las plantas trepadoras en general.

\section{Materiales y Métodos}

Esta contribución se basa en numerosos viajes de estudio realizados a la Isla Martín García (Buenos Aires, Argentina) desde 2011 hasta la fecha, en distintas estaciones del año. Se estudiaron 30 individuos en el terreno, distribuidos en diferentes formaciones vegetales presentes en la isla: bosques higrófilos, arenales interiores y bosques asociados, y bosques mixtos, con elementos arbóreos nativos y exóticos (Hurrell, 2008). Se coleccionó material de referencia de 5 individuos distribuidos en las diferentes formaciones vegetales, que fue depositado en el Herbario LP.

Cada individuo evaluado fue documentado fotográficamente y se estudiaron: su tipo biológico, mecanismos de ascenso, mecanismos de expansión, patrón de ramificación y relaciones con los soportes (presencia-ausencia, distribución espacial, diámetro). Además, se revisaron materiales de colecciones correspondientes a la Isla Martín García previas al desarrollo de este estudio, en los herbarios LP, SI, BA y BAB, que se indican como material adicional examinado.

Material de referencia. ARGENTINA. Prov. Buenos Aires: Isla Martín García, arenal occidental, 19-XII-2011 Cabanillas 13 (LP); casa de bombas, bosque higrófilo, 19-XII-2011, Cabanillas 14 (LP); arenal central, 19-XII-2011, Cabanillas 15 (LP); arenal occidental, 17-XII-2012, Cabanillas \& Saenz 62 (LP); bosque mixto, 17-XII-2012, Cabanillas \& Saenz 66 (LP).

Material adicional examinado. ARGENTINA. Prov. Buenos Aires: Isla Martín García, 31-X-1920, Clos 949 (BAB); X-1923, Pérez Moreau (BA 7078); VI-1926, Molfino \& Clos 441 (BAB); 18-X-1930, Jussen (BA 10911); 28-IX-1939, Pérez Moreau (BA 33225, 33226); 25-X-1951, O. Boelcke 4990 (SI); borde de selva y arenal central, 12-XII-1992, Hurrell et al. 1407 (LP); arenal central, 8-X-1993, Hurrell et al. 1502 (SI); bosque xeromórfico, 8-X1993, Hurrell et al. 1503 (SI); camino al crematorio, 28-VII-1994, Hurrell et al. 1835 (SI); camino al tanque de combustible, 10-IX-1994, Hurrell et al. 1956 (SI); camino al arenal central, 11-IX-1994, Hurrell et al.1972 (LP); camino a la cantera, 11-IX1994, Hurrell et al.1977 (LP); arenal noroeste, 6-X1995, Múlgura et al. 1504 (SI); cerca de la casa de bombas, 14-XII-1995, Hurrell et al. 2555 (BAB).

\section{Resultados}

Los ejemplares estudiados de E. tweediana presentan el tipo biológico liana, es decir, una trepadora escandente leñosa (fide Cabanillas \& Hurrell, 2012). Según los estudios realizados, los individuos de esta especie desarrollan dos sistemas de ejes caulinares: epiclinos (trepadores) y rastreros (Fig. 1A).

Los extremos de los tallos epiclinos suelen estar recurvados a modo de ganchos que, cuando presentes, colaboran en la fijación a los soportes (Fig. 1E). Los tallos epiclinos verdes son flexuosos y crecen casi verticales, sosteniéndose unos con otros, de modo que conforman por sí mismos un entramado de ramas que envuelven a los soportes; por lo general tienen $c a .1 \mathrm{~cm}$ diám., aunque en 

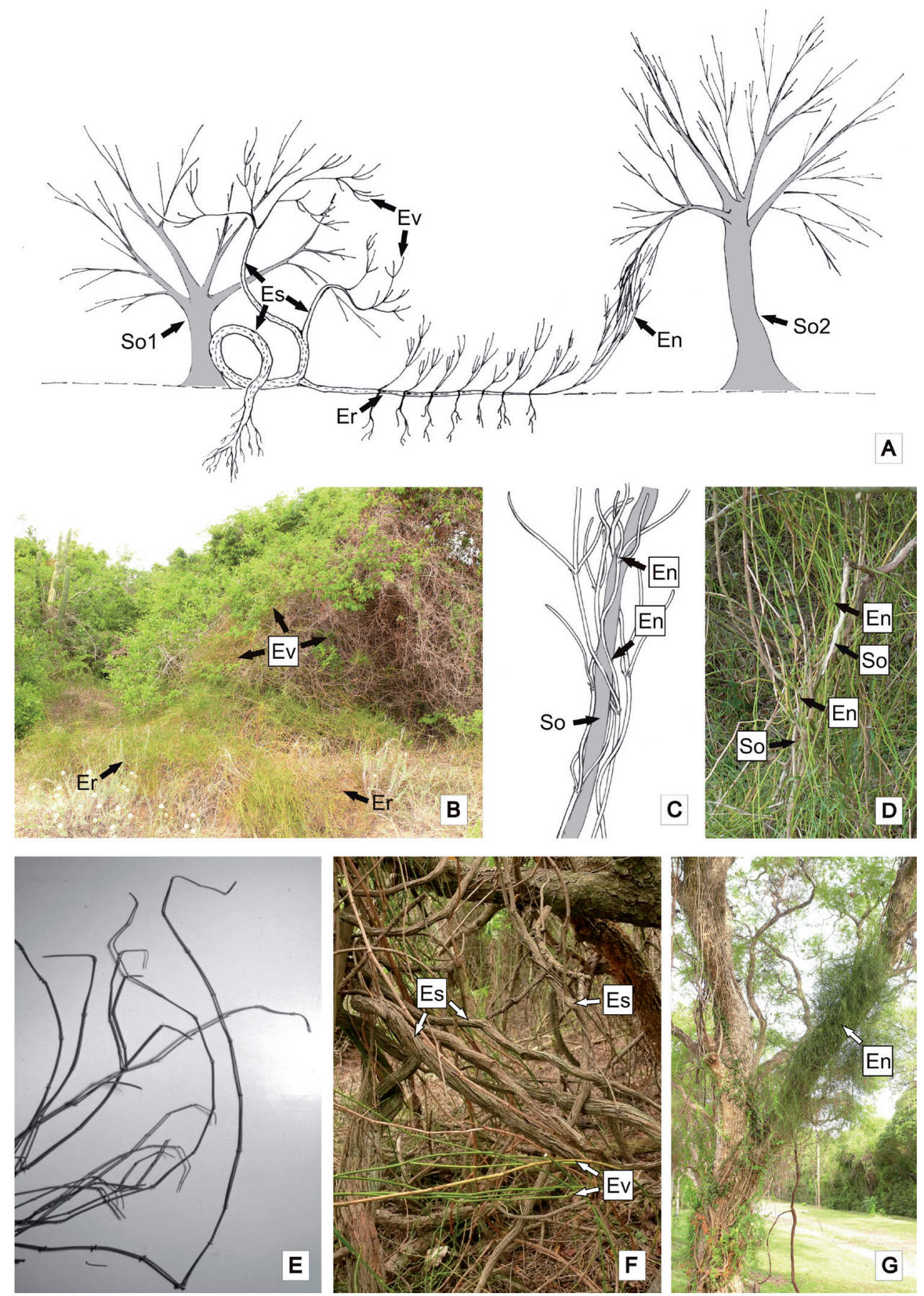

Fig. 1. Tipo biológico y estrategia de ascenso de Ephedra tweediana. A: Esquema del tipo biológico. B: Mantos conformados por ejes rastreros y por ejes trepadores. C, D: Estrategia de ascenso: entramado caulinar. E: Extremos de los ejes verdes recurvados a modo de ganchos. F: Aspecto de ejes trepadores verdes y sarmentosos. G: Ejes entramados sobre una rama gruesa del soporte. Abreviaturas: En entramado caulinar; Er ejes rastreros; Es ejes trepadores sarmentosos; Ev ejes trepadores verdes; So soporte (Daniel Bazzano: fotos B, D, F, G; Pablo Cabanillas: foto E y esquemas). 
algunos casos llegan a los $40 \mathrm{~cm}$ diám. (Fig. 1C, D, G). Por medio de este mecanismo, la planta crece sobre el soporte alcanzando hasta 15 metros de altura sobre el mismo. Estos ejes conforman vistosos mantos sobre los soportes, especialmente cuando presentan sus conos microsporagiados (amarillos) o megasporangiados (rojos) (Fig. 1B).

Los tallos epiclinos leñosos (sarmentosos) son muy retorcidos y mantienen una relación estrecha con el soporte aunque no se encuentren fijos al mismo (Fig. 1G). Alcanzan $10 \mathrm{~cm}$ diám. y varias decenas de metros de longitud sobre los soportes.

Los ejes rastreros son leñosos, superficiales o subsuperficiales, con nudos enraizantes de los que surgen nuevos vástagos profusamente ramificados, que crecen casi verticales apoyados unos en otros, alcanzando $60 \mathrm{~cm}$ alt. Estos ejes pueden devenir trepadores si contactan con un soporte. En ocasiones, los ejes rastreros conforman mantos que cubren varias decenas de metros cuadrados, como ocurre en los arenales interiores de Martín García (Fig. 1B).

Se observó un mayor desarrollo de ejes rastreros en áreas con escasa cubierta vegetal, en especial, en los arenales. La altura máxima alcanzada fue mayor en los bosques (15 m alt.). Asimismo, no se observaron diferencias cualitativas en cuanto al tipo biológico, mecanismos de ascenso y expansión de E. tweediana entre las diferentes formaciones vegetales.

\section{Discusión y Conclusiones}

La estrategia de ascenso de E. tweediana consiste en una especialización de los vástagos, en la cual las ramas jóvenes (que consideradas de modo individual podrían considerarse "apoyantes"), conforman un entramado que envuelve los soportes. Así, la única estrategia de ascenso se encuentra en las partes verdes. Es una situación similar a la que ocurre en muchas trepadoras que presentan zarcillos enroscantes, en las que el sostén de la planta es realizado sólo por las partes verdes. A diferencia de los tallos volubles que, de forma individual, se enroscan al soporte, los tallos jóvenes de $E$. tweediana sólo se encaraman al soporte en forma conjunta, como resultado de su propio desarrollo.

Podranea ricasoliana (Tanfani) Sprague (Bignoniaceae), presenta una estrategia de ascenso similar, considerada poco común y denominada "entrelazado" o "entretejido" (Menninger, 1970; Hurrell et al., 2012a). Los ejes trepadores de esta especie crecen alternativamente hacia la luz y hacia la sombra, de modo que cada uno de ellos se entrelaza con el soporte. "Entrelazar", según el diccionario de la Real Academia Española, significa 'enlazar algo con otra cosa' (para este caso, con el soporte), por lo cual el término se ajusta al caso de P. ricasoliana. En cambio, los ejes de E. tweediana conforman una matriz de ejes entramados en torno al soporte: no hay entrelazado con el soporte, sino un entramado caulinar que se origina a partir del patrón de ramificación de la propia trepadora alrededor del soporte. "Entramado", según el mismo diccionario, significa "conjunto de tiras de material flexible que se cruzan entre sí", y resulta adecuado para describir el caso en estudio. El hecho de que los tallos se "cruzan entre sí" permite distinguir claramente esta estrategia de ascenso de la de $P$. ricasoliana, cuyos ejes se entrelazan con los soportes. Esta peculiaridad observada en $E$. tweediana no se halla registrada en la literatura.

Los mecanismos de ascenso especializados son aquellos en los que se establece una estrecha relación con el soporte, como los tallos volubles, zarcillos y pecíolos prensiles que envuelven el soporte, o bien, los zarcillos uncinados y raíces aéreas que se adhieren al sostén. Los mecanismos no especializados, en cambio, implican estructuras que no abrazan ni se adhieren, pero aumentan la fricción de la planta contra la superficie del soporte, evitando que se deslice y caiga por su propio peso, como la disposición opuesta de las hojas, nudos engrosados, espinas, aguijones o pelos rígidos (Cabanillas \& Hurrell, 2012). Según este esquema, la estrategia de entramado caulinar característica de $E$. tweediana se define como un mecanismo de ascenso especializado.

E. tweediana ha sido generalmente considerada como un arbusto trepador apoyante (Hunziker, 1949, 1969, 1995; Hunziker \& Novara, 1998; Ferrucci et al., 2002), y en ocasiones, como una liana (Giehl, 2012). La designación de "arbusto" hace referencia a su comparación con los tipos biológicos de plantas erectas leñosas (lo cual no siempre es explícito y, por ende, puede inducir a error en la asignación de la forma de vida). Por otro lado, el término "apoyante" se refiere usualmente a las plantas trepadoras que no presentan mecanismos de ascenso especializados. Las "lianas", por su 


\section{P. A. Cabanillas et al.- Entramado caulinar en Ephedra tweediana}

parte, son plantas leñosas escandentes, es decir, presentan estrategias de ascenso especializadas (Hurrell, 2006; Cabanillas \& Hurrell, 2012).

Se concluye, por tanto, que E. tweediana es una liana con un doble sistema de ejes caulinares: rastreros y epiclinos (trepadores), bien representado en lianas de diversas familias de Angiospermas (Darwin, 1875; Putz \& Mooney, 1991; Hurrell et al., 2011, 2012a, b). Este sistema doble se describe por primera vez para E. tweediana.

\section{Agradecimientos}

Los autores desean expresar su agradecimiento al Prof. Emilio Ulibarri, Daniel Bazzano y Fernando Buet Constantino por su valiosa colaboración en los trabajos de campo.

\section{Bibliografía}

CABANILlAS, P. A. \& J. A. HURRELL. 2012. Plantas trepadoras: tipo biológico y clasificación. Rev. $\mathrm{Ci}$. Morfol. (La Plata) 14: 1-15.

CARLQUIST, S. 1996. Wood, bark, and stem anatomy of Gnetales: a summary. Int. J. Plant Sci. 157 (Suppl.): S58-S76.

CHASE, M. W. \& J. L. REVEAL. 2009. A phylogenetic classification of the land plants to accompany APG III. Bot. J. Linn. Soc. 161: 122-127.

CHRISTENHUSZ, M. J. M., J. L. REVEAL, A. FARJON, M. F. GARDNER, R. R. MILL \& M. W. CHASE. 2011. A new classification and linear sequence of extant gymnosperms. Phytotaxa 19: 55-70.

CREMERS, G.1973. Architecture de quelques lianes d'Afrique Tropicale 1. Candollea 28: 249-280.

CREMERS, G. 1974. Architecture de quelques lianes d'Afrique Tropicale 2. Candollea 29: 57-110.

DARWIN, C. 1875. On the movements and habits of climbing plants. Bot. J. Linn. Soc. 9: 1-118.

FERRUCCI, M. S., S. A. CÁCERES MORAL \& M. GALBANY CASALS. 2002. Las plantas trepadoras. En Arbo, M. M. \& S. G. Tressens (eds.), Flora del Iberá, pp. 111-153. EUDENE, Corrientes.

FONT QUER, P. 1993. Diccionario de Botánica. 1244 pp. Ed. Labor, Barcelona.

GIEHL, E. L. (coord.). 2012. Ephedra. En Flora Digital do Rio Grande do Sul e Santa Catarina. Disponible: http//ufrga.br./flora digital [Consulta: 12-XII-2012].

HUNZIKER, J. H. 1949. Sinopsis de las especies argentinas del género Ephedra. Lilloa 17: 147-174.

HUNZIKER, J. H. 1969. Ephedraceae. En Cabrera, A. L. (ed.), Fl. Prov. Buenos Aires, Colecc. Ci. Inst. Nac. Tecnol. Agropecu. 4: 267-271.

HUNZIKER, J. H. 1995. Ephedraceae. En Hunziker, A. T. (ed.), Fl. Fanerog. Argent. 4 (7a): 15-23. ProfloraCONICET. Córdoba.

HUNZIKER, J. H. \& L. J. NOVARA. 1998. Ephedraceae. En Novara, L. J. (ed.), Fl. Valle de Lerma. Aportes Bot. Salta 5: 1-14.

HURRELL, J. A. 2006. Introducción. En Hurrell J. A. (ed,), Biota Rioplatense V. Plantas trepadoras nativas y exóticas. pp. 16-38. Editorial LOLA. Buenos Aires.

HURRELL, J. A. 2008. Área de estudio. En Hurrell J. A. (ed.), Flora Rioplatense 3: 11-13. Editorial LOLA, Buenos Aires.

HURRELL, J.A., P.A. CABANILLAS \& G. DELUCCHI. 2011. Wisteria sinensis (Leguminosae) adventicia en la Argentina. Primer registro y mecanismos de expansión. Rev. Mus. Argentino Cienc. Nat., n.s., 13: 125-130.

HURRELL, J. A., P. A. CABANILlAS, F. BUET COSTANTINO \& G. DELUCCHI. 2012a. Bignoniaceae adventicias en la Argentina. Primera cita de Podranea ricasoliana y nuevos registros de Campsis radicans. Rev. Mus. Argentino Cienc. Nat., n.s., 14: 15-22.

HURRELL, J. A, G. DELUCCHI \& P.A. CABANILLAS. 2012b. Primera cita de Parthenocissus tricuspidata y nuevo registro de $P$. quinquefolia (Vitaceae) adventicias en la Argentina. Rev. Mus. Argent. Cienc. Nat., n.s., 14: 235-242.

ICKERT-BOND, S. M. \& M. F. WOJCIECHOWSKI. 2004. Phylogenetic Relationships in Ephedra (Gnetales): Evidence from Nuclear and Chloroplast DNA Sequence Data. Systematic Botany 29: 834 849.

INSTITUTO DE BOTÁNICA DARWINION. 2012. Flora del cono sur. Disponible: http://www2.darwin. edu.ar/Proyectos/FloraArgentina/FA.asp [Consulta: 12-XII-2012].

MENNINGER, E. A. 1970. Flowering vines of the world: an encyclopedia of climbing plants. $410 \mathrm{pp}$. Hearthside Press Incorporated, New York.

PUTZ, F. E. \& H. A. MOONEY (eds.). 1991. Biology of vines. 535 pp. Cambridge University Press, Cambridge.

SOUZA, V. C. 2012. Gimnospermas. En Lista de Espécies da Flora do Brasil. Jardim Botânico do Rio de Janeiro. Disponible: http://floradobrasil.jbrj.gov. br/2012/FB035363 [Consulta: 12-XII-2012].

Recibido el 7 de febrero de 2013, aceptado el 13 de junio de 2013 . 
